\title{
Mass and Ion Transport in Ketones and Ketone Electrolytes: Comparison with Acetate Systems
}

\author{
Dharshani N. Bopege • Matt Petrowsky • Matthew B. Johnson • \\ Roger Frech
}

Received: 8 June 2012/ Accepted: 3 August 2012/Published online: 19 March 2013

(C) The Author(s) 2013. This article is published with open access at Springerlink.com

\begin{abstract}
Self-diffusion coefficient measurements were performed for pure $n$-alkyl ketone liquids using the pulsed field gradient NMR spin-echo technique. Ionic conductivities and dielectric constants of $0.0055 \mathrm{~mol} \cdot \mathrm{L}^{-1}$ tetrabutylammonium trifluoromethanesulfonate in 2-pentanone, 2-hexanone, 2-heptanone, 2-octanone, 2-nonanone, and 2-decanone were also measured. The temperature-dependent conductivities and diffusion coefficients over the range $5-80{ }^{\circ} \mathrm{C}$ can be described using the compensated Arrhenius formalism. Compensated Arrhenius equation plots were used to calculate the average activation energy for both sets of data. The average activation energy from conductivity data is approximately equal to that from diffusion data. The data for the pure ketones and ketone-based electrolytes are compared with analogous data for pure $n$-alkyl acetates and $n$-alkyl acetate-based electrolytes.
\end{abstract}

Keywords Ionic conductivity · Diffusion coefficient - Compensated Arrhenius · Dielectric constant $\cdot$ Ketone electrolyte $\cdot$ Acetate electrolyte

\section{Introduction}

Mass and charge transport in organic liquid electrolytes have garnered interest due to the use of these electrolytes in electrochemical devices. Ionic conductivities and self-diffusion coefficients are important measures of transport phenomena, although these data are conventionally described with viscosity-related models [1-3] that often predict results that do not agree with experiment [4-6]. Recently, mass and charge transport have been viewed

Electronic supplementary material The online version of this article (doi:10.1007/s10953-013-9983-z) contains supplementary material, which is available to authorized users.

D. N. Bopege - M. B. Johnson

Homer L. Dodge Department of Physics and Astronomy, University of Oklahoma, 440 W. Brooks St., Norman, OK 73019, USA

M. Petrowsky $\cdot$ R. Frech $(\bowtie)$

Department of Chemistry and Biochemistry, University of Oklahoma, 101 Stephenson Parkway,

Norman, OK 73019, USA

e-mail: rfrech@ou.edu 
from an entirely different perspective [7,8], by postulating that the conductivity and diffusion coefficient assume an Arrhenius-like expression with static dielectric constant $\left(\varepsilon_{\mathrm{s}}\right)$ dependence in the exponential prefactor:

$$
\begin{gathered}
\sigma\left(T, \varepsilon_{\mathrm{s}}\right)=\sigma_{0}\left\{\varepsilon_{\mathrm{s}}(T)\right\} \exp \left(-E_{a} / R T\right) \\
D\left(T, \varepsilon_{\mathrm{s}}\right)=D_{0}\left\{\varepsilon_{\mathrm{s}}(T)\right\} \exp \left(-E_{a} / R T\right)
\end{gathered}
$$

Here $\sigma\left(T, \varepsilon_{\mathrm{s}}\right)$ is the ionic conductivity, $D\left(T, \varepsilon_{\mathrm{s}}\right)$ is the self-diffusion coefficient, $\sigma_{0}\left\{\varepsilon_{\mathrm{s}}(T)\right\}$ and $D_{0}\left\{\varepsilon_{\mathrm{s}}(T)\right\}$ are the exponential prefactors for conductivity and diffusion, respectively. In addition, $E_{\mathrm{a}}$ is the activation energy and $T$ is the temperature.

The prefactors in Eqs. 1 and 2 are temperature dependent due to the inherent temperature dependence of the dielectric constant. The dielectric constant dependence can be canceled by using a scaling procedure that has been previously described in detail [7-9]. The scaling procedure consists of dividing the temperature-dependent conductivities (or diffusion coefficients) by conductivities (or diffusion coefficients) at a reference temperature $T_{\mathrm{r}}$. The two quantities are chosen such that the temperature-dependent quantity and the reference quantity have the same value of $\varepsilon_{\mathrm{s}}$. This scaling results in compensated Arrhenius equations (CAE) for conductivity and diffusion:

$$
\begin{aligned}
& \ln \left(\frac{\sigma\left(T, \varepsilon_{\mathrm{s}}\right)}{\sigma_{\mathrm{r}}\left(T_{\mathrm{r}}, \varepsilon_{\mathrm{s}}\right)}\right)=-\frac{E_{\mathrm{a}}}{R T}+\frac{E_{\mathrm{a}}}{R T_{\mathrm{r}}} \\
& \ln \left(\frac{D\left(T, \varepsilon_{\mathrm{s}}\right)}{D_{\mathrm{r}}\left(T_{\mathrm{r}}, \varepsilon_{s}\right)}\right)=-\frac{E_{\mathrm{a}}}{R T}+\frac{E_{\mathrm{a}}}{R T_{\mathrm{r}}}
\end{aligned}
$$

The activation energies for conductivity and diffusion can be calculated from either the slope or intercept of Eqs. 3 and 4, respectively. Equations 1-4 and the postulates therein constitute the compensated Arrhenius formalism (CAF).

In this study, activation energies are reported for diffusion data of pure ketones and conductivity data of $0.0055 \mathrm{~mol} \cdot \mathrm{L}^{-1}$ tetrabutylammonium trifluoromethanesulfonate (TbaTf)-ketone solutions. We have previously reported activation energies from conductivity data for dilute ketone electrolytes that focused on short chain ketones [7]. The ketones studied here include 2-pentanone, 2-hexanone, 2-heptanone, 2-octanone, 2-nonanone and 2-decanone. This paper also compares the conductivity and diffusion data in ketones with analogous data from dilute acetate electrolytes and pure acetates [10]. Ketones and acetates are both aprotic solvents with carbonyl groups. However, the permittivity of ketones is significantly higher than that for acetates. Because the dielectric constant plays a prominent role in transport phenomena, it is important to compare conductivity and diffusion data between ketones and acetates.

\section{Experimental}

\subsection{Materials}

2-Pentanone (99+\%), 2-octanone (98\%), and 2-decanone (97\%) were obtained from Alfa Aesar, while 2-hexanone (98\% reagent grade), 2-heptanone (99\% reagent plus grade), and 2-nonanone (99+ \% reagent plus grade) were from Aldrich. TbaTf (99\%) was purchased from Aldrich and used as received. The samples were prepared in a glove box under a nitrogen atmosphere ( $\leq 1 \mathrm{ppm} \mathrm{H}_{2} \mathrm{O}$ and approximate temperature $25^{\circ} \mathrm{C}$ ). TbaTf 
was dissolved in the appropriate amount of ketone to make the $0.0055 \mathrm{~mol} \cdot \mathrm{L}^{-1}$ solution, and then stirred for $24 \mathrm{~h}$ before use.

\subsection{Measurements}

Each sample was injected into an Agilent 16452A liquid test fixture with a $2 \mathrm{~mm}$ spacer and immersed in an oil bath whose temperature was controlled from 5 to $85^{\circ} \mathrm{C}$, in increments of $10{ }^{\circ} \mathrm{C}$, with a Huber Ministat 125 . The conductance and capacitance were measured at each temperature (within $0.3{ }^{\circ} \mathrm{C}$ of the set temperature) over the frequency range $1 \mathrm{kHz}$ to $13 \mathrm{MHz}$ using a $\mathrm{HP} 4192 \mathrm{~A}$ impedance analyzer. The conductivity, $\sigma$, was calculated from the measured conductance, $G$, through the equation $\sigma=L G A^{-1}$, where $L$ is the electrode gap and $A$ is the electrode area. The cell constant calculated from the cell geometry is $0.0176 \mathrm{~cm}^{-1}$. The static dielectric constant $\varepsilon_{\mathrm{s}}$ was calculated from the measured capacitance $C$ using the equation $\varepsilon_{\mathrm{s}}=\alpha C C_{0}^{-1}$, where $\alpha$ is a variable to account for stray capacitance and $C_{0}$ is the atmospheric capacitance.

A Varian VNMRS $400 \mathrm{MHz}$ NMR spectrometer was utilized to measure self diffusion coefficients from 5 to $80{ }^{\circ} \mathrm{C}$. The Larmor frequency for protons was $399.870 \mathrm{MHz}$ using an Auto-X-dual broad band $(5 \mathrm{~mm})$ probe. The Stejskal-Tanner pulsed field gradient NMR spin-echo sequence was used for the diffusion measurements [11]. At each temperature, the gradient field strength was arrayed from 6 to $62 \mathrm{G} \cdot \mathrm{cm}^{-1}$ and the integrated intensity of each attenuated signal was calculated. The diffusion coefficient was calculated from the slope of the plot $\ln$ (intensity) versus square of the gradient strength. The temperature was controlled using an FTS XR401 air-jet regulator.

\subsection{Data Analysis}

A high degree of linearity was observed for both simple Arrhenius and compensated Arrhenius plots for the self-diffusion coefficient data of pure ketones (data are not shown). Table 1 summarizes the compensated Arrhenius $E_{\mathrm{a}}$ values at five different reference temperatures $\left(25,35,45,55,65^{\circ} \mathrm{C}\right)$ for pure 2-hexanone, 2-heptanone, and 2-nonanone. It is important to note that the value of $E_{\mathrm{a}}$ does not significantly depend on the choice of the reference temperature.

Table 1 Activation energies for pure ketones from compensated and simple Arrhenius plots of diffusion data

\begin{tabular}{|c|c|c|c|c|c|}
\hline \multirow[b]{2}{*}{ Ketone } & \multirow[b]{2}{*}{$T_{\mathrm{r}}\left({ }^{\circ} \mathrm{C}\right)$} & \multicolumn{2}{|c|}{$\mathrm{CAE} E_{\mathrm{a}} / \mathrm{kJ} \cdot \mathrm{mol}^{-1}$} & \multicolumn{2}{|c|}{ Simple Arrhenius $E_{\mathrm{a}} / \mathrm{kJ} \cdot \mathrm{mol}^{-1}$} \\
\hline & & Slope & Intercept & Ketone & Slope \\
\hline \multirow[t]{2}{*}{ 2-Hexanone } & 25 & $23.8 \pm 0.5$ & $23.9 \pm 0.5$ & 2-Hexanone & $15.2 \pm 0.2$ \\
\hline & 35 & $23.7 \pm 0.5$ & $23.8 \pm 0.5$ & 2-Heptanone & $15.2 \pm 0.6$ \\
\hline \multirow[t]{5}{*}{ 2-Heptanone } & 25 & $23.8 \pm 0.9$ & $24.0 \pm 0.8$ & 2-Octanone & $16.1 \pm 0.7$ \\
\hline & 35 & $23.8 \pm 0.9$ & $24.0 \pm 0.9$ & 2-Nonanone & $15.1 \pm 0.1$ \\
\hline & 45 & $23.8 \pm 0.9$ & $24.0 \pm 0.9$ & 2-Decanone & $15.7 \pm 0.2$ \\
\hline & 55 & $23.9 \pm 0.9$ & $24.0 \pm 0.9$ & & \\
\hline & 65 & $23.9 \pm 0.9$ & $23.9 \pm 0.9$ & & \\
\hline 2-Nonanone & 65 & $24.0 \pm 0.7$ & $23.9 \pm 0.7$ & & \\
\hline
\end{tabular}


The average CAE activation energy from the data in Table 1 is $(23.9 \pm 0.8) \mathrm{kJ} \cdot \mathrm{mol}^{-1}$. Table 1 also lists the simple Arrhenius $E_{\mathrm{a}}$ values for each ketone obtained from a plot of $\mathrm{ln}$ $D$ versus $1 / T$. The $E_{\mathrm{a}}$ value obtained from the simple Arrhenius plot is lower than the corresponding CAE activation energies, which is a trend that has been observed in other systems [8-10]. Figure 1a plots self-diffusion coefficients versus static dielectric constant for pure ketones. Six well-separated curves are observed, one each for the temperature dependent data of each ketone.

The exponential prefactors, $D_{0}$, were calculated from Eq. 2 by dividing the temperature dependent diffusion coefficients by the Boltzmann factor, $\exp \left(-E_{\mathrm{a}} / R T\right)$. The plot of exponential prefactor versus dielectric constant yields a single master curve in Fig. $1 \mathrm{~b}$. This single master curve can be observed only for a narrow range of $E_{\mathrm{a}}$ values $\left(22.5-25.5 \mathrm{~kJ} \cdot \mathrm{mol}^{-1}\right)$. The CAE gives $E_{\mathrm{a}}$ values that are within this range, while the simple Arrhenius plots yield activation energies that do not result in a master curve.

For conductivity data of $0.0055 \mathrm{~mol} \cdot \mathrm{L}^{-1}$ TbaTf-2-ketone systems, CAE plots exhibit linear behavior while simple Arrhenius plots are approximately linear but do show slight curvature (data not shown). The resulting CAE and simple Arrhenius $E_{\mathrm{a}}$ values are reported in Table 2. Similar to the diffusion data, simple Arrhenius $E_{\text {a }}$ values are lower than those from the CAE. The average CAE activation energy from the data in Table 2 is $(24.1 \pm 0.8) \mathrm{kJ} \cdot \mathrm{mol}^{-1}$; this value was utilized to determine the conductivity exponential prefactors by dividing the temperature-dependent conductivities in Eq. 1 by the Boltzmann factor.

Temperature-dependent ionic conductivities are plotted against temperature-dependent dielectric constants in Fig. $2 \mathrm{a}$ for the $0.0055 \mathrm{~mol} \cdot \mathrm{L}^{-1}$ TbaTf-ketone data. Six distinct curves are observed, one for each ketone electrolyte solution. However, a single master curve is observed when the exponential prefactors are plotted against the static dielectric constant, as shown in Fig. 2b. A master curve is only observed for $E_{\mathrm{a}}$ values in the narrow range from 22 to $27 \mathrm{~kJ} \cdot \mathrm{mol}^{-1}$. Similar to the diffusion results, the CAE activation energies result in a master curve, while those from the simple Arrhenius equation do not.
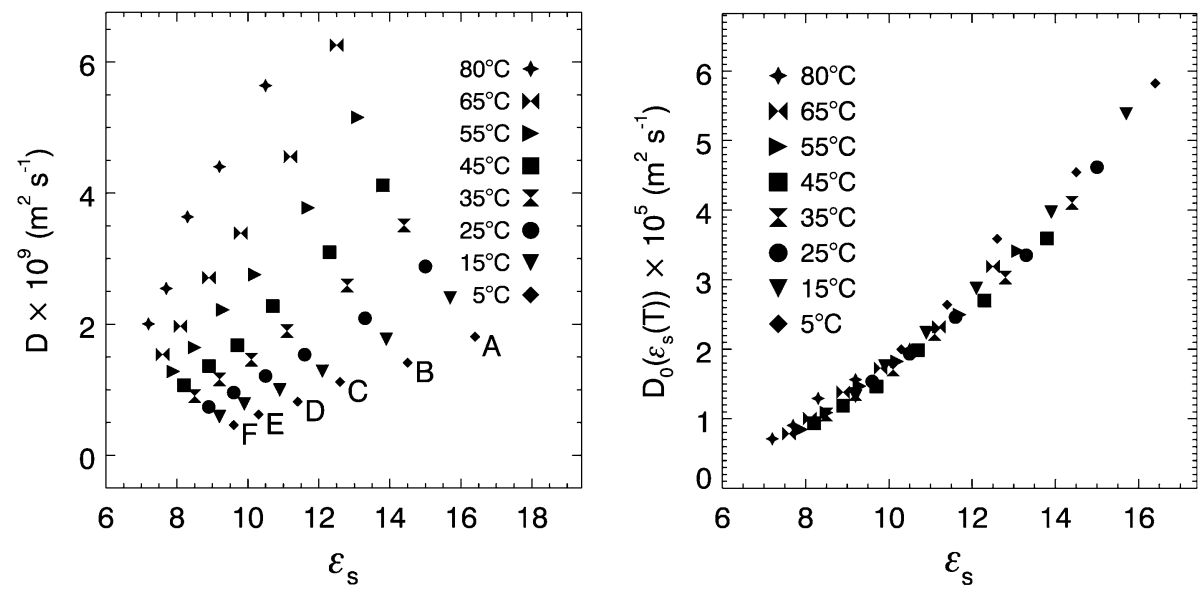

Fig. 1 Left: Self-diffusion coefficients of pure ketones versus static dielectric constant from 5 to $80{ }^{\circ} \mathrm{C}$ for: $(A)$ 2-pentanone, $(B)$ 2-hexanone, $(C)$ 2-heptanone, $(D)$ 2-octanone, $(E)$ 2-nonanone, and $(F)$ 2-decanone. Right: Exponential prefactor versus dielectric constant for the diffusion data of pure ketones using $E_{\mathrm{a}}=23.9 \mathrm{~kJ} \cdot \mathrm{mol}^{-1}$ 
Table 2 Activation energies from compensated and simple Arrhenius plots resulting from conductivity data for $0.0055 \mathrm{~mol} \cdot \mathrm{L}^{-1}$ TbaTf-2-ketones

\begin{tabular}{|c|c|c|c|c|c|}
\hline \multirow[b]{2}{*}{ Ketone } & \multirow[b]{2}{*}{$T_{\mathrm{r}}\left({ }^{\circ} \mathrm{C}\right)$} & \multicolumn{2}{|c|}{$\mathrm{CAE} E_{\mathrm{a}} / \mathrm{kJ} \cdot \mathrm{mol}^{-1}$} & \multicolumn{2}{|c|}{ Simple Arrhenius $E_{\mathrm{a}} / \mathrm{kJ} \cdot \mathrm{mol}^{-1}$} \\
\hline & & Slope & Intercept & Ketone & Slope \\
\hline \multirow[t]{2}{*}{ 2-Hexanone } & 25 & $23.1 \pm 0.6$ & $23.2 \pm 0.6$ & 2-Pentanone & $5.0 \pm 0.1$ \\
\hline & 35 & $22.7 \pm 0.5$ & $22.8 \pm 0.5$ & 2-Hexanone & $5.2 \pm 0.1$ \\
\hline \multirow[t]{4}{*}{ 2-Heptanone } & 25 & $23.9 \pm 1.0$ & $24.1 \pm 1.0$ & 2-Heptanone & $5.6 \pm 0.2$ \\
\hline & 35 & $24.1 \pm 0.9$ & $24.3 \pm 0.9$ & 2-Octanone & $6.3 \pm 0.2$ \\
\hline & 45 & $23.4 \pm 0.8$ & $23.6 \pm 0.8$ & 2-Nonanone & $7.2 \pm 0.2$ \\
\hline & 55 & $23.0 \pm 0.5$ & $23.2 \pm 0.6$ & 2-Decanone & $8.5 \pm 0.2$ \\
\hline \multirow[t]{3}{*}{ 2-Octanone } & 45 & $25 \pm 1$ & $25 \pm 1$ & & \\
\hline & 55 & $25.3 \pm 0.9$ & $25.3 \pm 0.9$ & & \\
\hline & 65 & $24.8 \pm 0.9$ & $24.7 \pm 0.9$ & & \\
\hline 2-Nonanone & 80 & $25.4 \pm 0.8$ & $25.1 \pm 0.9$ & & \\
\hline
\end{tabular}
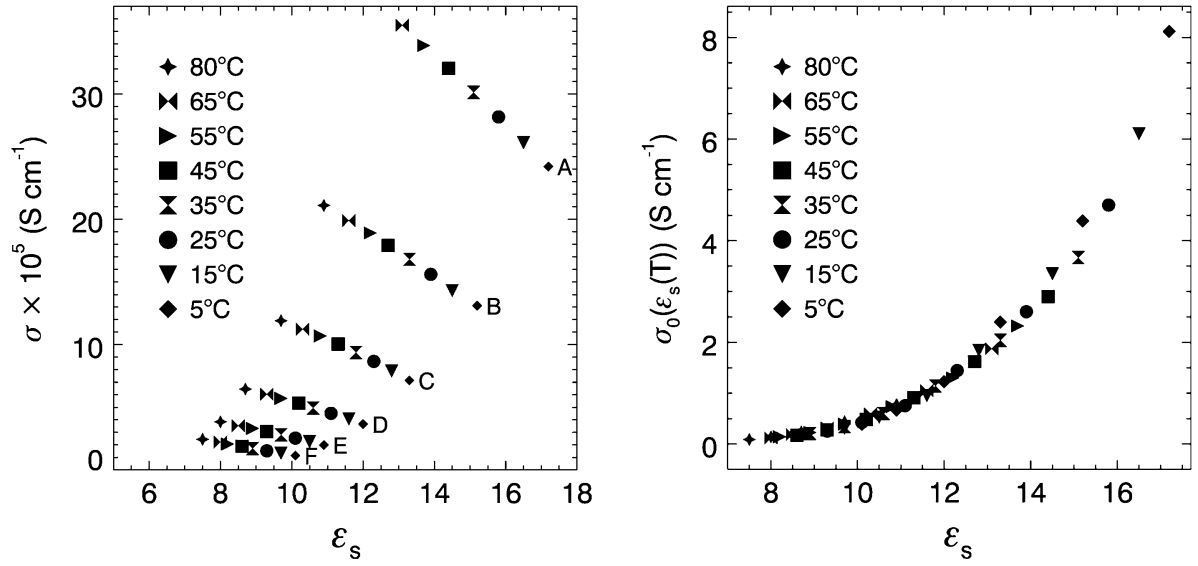

Fig. 2 Left: Conductivity versus static dielectric constant for $0.0055 \mathrm{~mol} \cdot \mathrm{L}^{-1}$ TbaTf-ketone solutions of $(A)$ 2-pentanone, $(B)$ 2-hexanone, $(C)$ 2-heptanone, $(D)$ 2-octanone, $(E)$ 2-nonanone, and $(F)$ 2-decanone over the temperature range 5 to $80^{\circ} \mathrm{C}$. Right: Exponential prefactor versus the dielectric constant for $0.0055 \mathrm{~mol} \cdot \mathrm{L}^{-1} \mathrm{TbaTf}-\mathrm{ketone}$ solutions using $E_{\mathrm{a}}=24.1 \mathrm{~kJ} \cdot \mathrm{mol}^{-1}$

A comparison of the conductivities of the $0.0055 \mathrm{~mol} \cdot \mathrm{L}^{-1}$ TbaTf-ketone solutions in the present study with the conductivities of $0.0055 \mathrm{~mol} \cdot \mathrm{L}^{-1} \mathrm{TbaTf}-$ acetate solutions from an earlier study [10] is shown in Fig. 3a. The conductivities of both ketone and acetate solutions increase with increasing temperature and decreasing alkyl chain length as expected. However, despite sharing commonalities in their chemical structures (the carbonyl oxygen common to both presumably coordinates the cation in electrolyte solutions), the conductivities of the TbaTf-ketone solutions are significantly higher than those of the TbaTf-acetate solutions. What is the origin of this difference?

The CAF provides important insight into this difference. In Eq. 1 there are two factors that control the conductivity: the exponential prefactor $\sigma_{0}$ and the Boltzmann factor $\exp \left(-E_{\mathrm{a}} / R T\right)$. Figure $4 \mathrm{a}$ shows that the exponential prefactors calculated from the conductivity data are very similar for the acetates and ketones. By way of comparison, a 

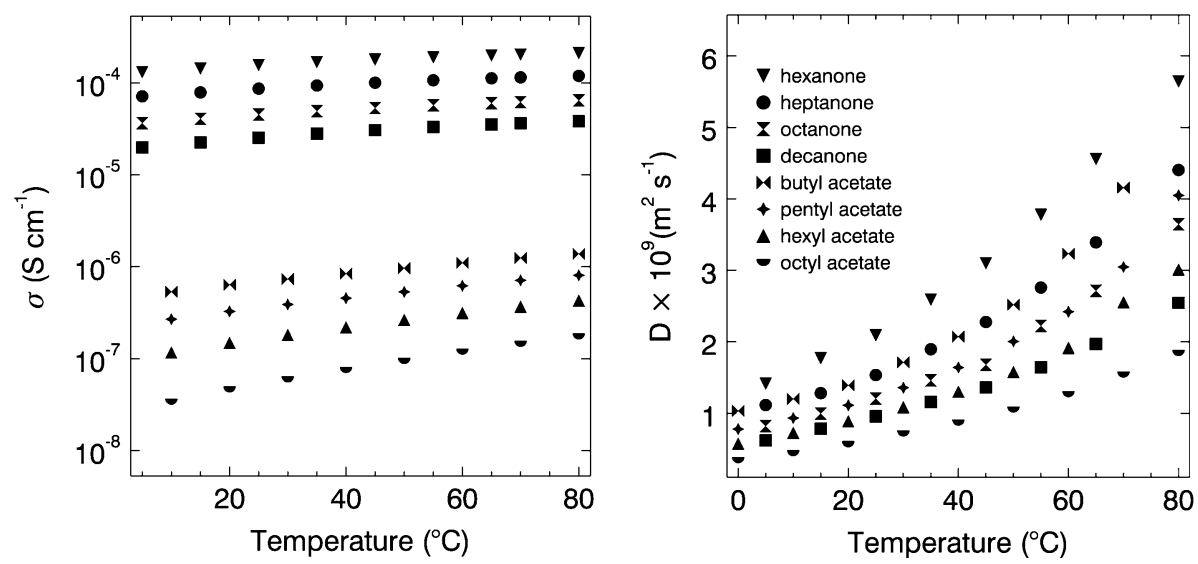

Fig. 3 Left: Conductivity versus temperature for $0.0055 \mathrm{~mol} \cdot \mathrm{L}^{-1}$ TbaTf-2-ketone solutions and $0.0055 \mathrm{~mol} \cdot \mathrm{L}^{-1}$ TbaTf- $n$-acetate solutions from 5 to $80^{\circ} \mathrm{C}$. Right: Self-diffusion coefficient versus temperature for pure ketone and pure acetate solvents

$0.004 \mathrm{~mol} \cdot \mathrm{L}^{-1}$ solution of TbaTf in 1-hexanol has $\sigma_{0}=1.10 \times 10^{4} \mathrm{~S} \cdot \mathrm{cm}^{-1}$ at $5{ }^{\circ} \mathrm{C}$ $\left(\varepsilon_{\mathrm{s}}=15.5\right)$ and $\sigma_{0}=2.70 \times 10^{2} \mathrm{~S} \cdot \mathrm{cm}^{-1}$ at $85^{\circ} \mathrm{C}\left(\varepsilon_{\mathrm{s}}=8.0\right)$ (unpublished data).

In a previous study, we found that the average CAE conductivity activation energy for a series of $0.0055 \mathrm{~mol} \cdot \mathrm{L}^{-1}$ TbaTf-acetate solutions was $36.5 \mathrm{~kJ} \cdot \mathrm{mol}^{-1}$, which is significantly higher than the value of $24.1 \mathrm{~kJ} \cdot \mathrm{mol}^{-1}$ for the $0.0055 \mathrm{~mol} \cdot \mathrm{L}^{-1} \mathrm{TbaTf}-\mathrm{ketone}$ solutions in the present study. Since the values of the prefactors are so similar, the higher conductivities of the ketone electrolytes originate in their markedly lower activation energies compared with the acetate electrolytes.
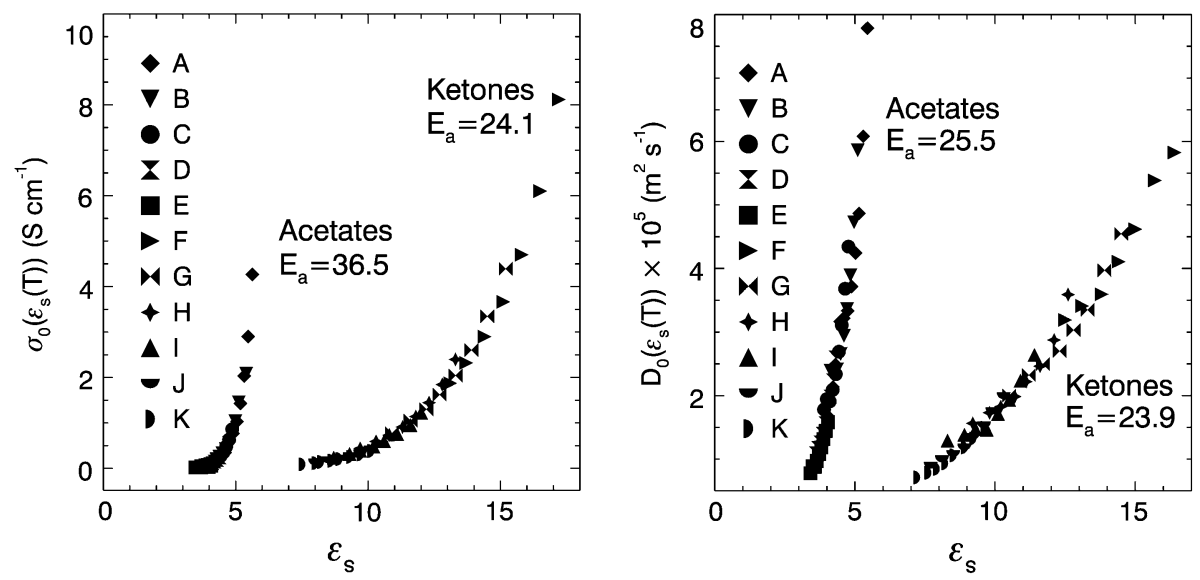

Fig. 4 Left: Exponential prefactor versus dielectric constant for the conductivity data of $0.0055 \mathrm{~mol} \cdot \mathrm{L}^{-1}$ TbaTf-acetates and $0.0055 \mathrm{~mol} \cdot \mathrm{L}^{-1}$ TbaTf-ketones. Right: Exponential prefactor versus dielectric constant for the diffusion data of pure acetates and pure ketones. The solvents are designated by: $(A)$ butyl acetate, $(B)$ pentyl acetate, $(C)$ hexyl acetate, $(D)$ octyl acetate, $(E)$ decyl acetate, $(F)$ 2-pentanone, $(G)$ 2-hexanone, $(H)$ 2-heptanone, $(I)$ 2-octanone, $(J)$ 2-nonanone, $(K)$ 2-decanone. The units of $E_{\mathrm{a}}$ are $\mathrm{kJ} \cdot \mathrm{mol}^{-1}$ 
The conductivity of an electrolyte can be expressed as $\sigma=\Sigma_{i} n_{i} q_{i} \mu_{i}$, where the summation runs over all charged species in the system and $n_{i}$ is the number density of charge carriers of type $i$ with charge $q_{i}$ and mobility $\mu_{i}$. The conductivity difference between the ketone and acetate solutions cannot be due to the number density of ions since both electrolytes have the same salt concentration $\left(0.0055 \mathrm{~mol} \cdot \mathrm{L}^{-1}\right)$. Numerous studies of ionic association in various Tba salt solutions have shown that weak, solvent-separated ion pairing occurs [12-15]. However, the charge-protected Tba cation does not form discrete, spectroscopically observable ionic species such as are found in lithium and sodium salt solutions [16-18]. Consequently, TbaTf electrolytes consist only of spectroscopically "free" ions, and ion mobility must be the key factor that controls the conductivity in these electrolytes. Accordingly, we conclude that the ion mobilities in the ketone electrolytes are substantially higher than those in the acetate electrolytes.

In contrast to the conductivity data, diffusion coefficients are comparable between pure ketones and acetates for similar temperatures and chain lengths as shown in Fig. 3b. The average compensated Arrhenius diffusion activation energy for the pure ketones is $23.9 \mathrm{~kJ} \cdot \mathrm{mol}^{-1}$ and that for the pure acetates is $25.5 \mathrm{~kJ} \cdot \mathrm{mol}^{-1}$. The diffusion prefactors are also comparable between acetates and ketones as seen in Fig. 4b; therefore, it is not surprising that the values of the diffusion coefficients of the ketones and acetates are similar. Indeed the primary difference between these two solvent families is that the acetate prefactor master curve is horizontally shifted on the permittivity axis from the ketone curve for both conductivity and diffusion prefactors in Fig. 4. This shift is due to the difference in permittivity originating in the difference in dipole moments for these two solvent families. For example, the gas phase permanent moment of ethyl acetate $\left(\mathrm{C}_{4} \mathrm{H}_{8} \mathrm{O}_{2}\right)$ is $1.78 \mathrm{D}$ [19] while that of 2-butanone $\left(\mathrm{C}_{4} \mathrm{H}_{8} \mathrm{O}\right)$ is $2.78 \mathrm{D}$ [20].

The $E_{\mathrm{a}}$ for diffusion in pure ketones is approximately equivalent to the $E_{\mathrm{a}}$ for conductivity in the dilute TbaTf-ketone solutions as indicated in Fig. 4. However, the addition of a small amount of TbaTf to a pure acetate solvent increases the average activation energy from 25.5 to $36.5 \mathrm{~kJ} \mathrm{~mol}{ }^{-1}$ in the $0.0055 \mathrm{~mol} \cdot \mathrm{L}^{-1}$ solution. This difference may originate in the lower permittivities of the acetate systems that lead to stronger ion-solvent interactions and contribute in large part to the $10 \mathrm{~kJ} \cdot \mathrm{mol}^{-1}$ difference in their $E_{\mathrm{a}}$ values.

One of our previous CAF studies involved $0.0055 \mathrm{~mol} \cdot \mathrm{L}^{-1}$ TbaTf-ketone solution conductivity data that included the short chain ketones 2-butanone and acetone. This study resulted in an average activation energy of $\sim 16 \mathrm{~kJ} \cdot \mathrm{mol}^{-1}$ [7]. Numerous CAF studies of other solvent-based systems (alcohols, ketones, acetates, nitriles, acyclic carbonates) have led to the conclusion that including the shortest alkyl chain members of a solvent family in the CAF analysis produces activation energies that are somewhat lower than if these members are omitted. The present work gives an average conductivity activation energy of $24.1 \mathrm{~kJ} \cdot \mathrm{mol}^{-1}$ by leaving out the acetone and 2-butanone data. Further, the activation energies calculated here for 2-hexanone, 2-heptanone, 2-octanone, and 2-nonanone are remarkably close to each other as seen in Table 2 .

Acknowledgments We wish to thank the Army Research Office for support of this work through Grant No. W911NF-10-1-0437. We are grateful to Dr. Susan Nimmo for her guidance with the NMR diffusion measurements and acknowledge the National Science Foundation for its financial support of the NMR equipment (Grant No: CHE\#0639199). We thank the Johnson research group in the OU physics department, and especially Jeremy Jernigen, for their help with glovebox modifications. We appreciate the expertise of Chris Crowe in software development for the HP 4192A. 
Open Access This article is distributed under the terms of the Creative Commons Attribution License which permits any use, distribution, and reproduction in any medium, provided the original author(s) and the source are credited.

\section{References}

1. Walden, P.: Connection between viscosity and ionic velocity or rate of diffusion. Z. Elektrochem. Angew. Phys. Chem. 12, 77-78 (1906)

2. Bockris, J.O.M., Reddy, A.K.N.: Modern Electrochemistry, vol. 1. Plenum Press, New York (1998)

3. Levine, I.N.: Physical Chemistry, 6th edn. McGraw-Hill, New York (2009)

4. Longsworth, L.G., MacInnes, D.A.: Ion conductances in water-methanol mixtures. J. Phys. Chem. 43, 239-246 (1939)

5. Moreira, L., Leitao, R.E., Martins, F.: Solvent and temperature effects on ion association and mobility of 2,6-lutidinium chloride in non-aqueous solvents. Mol. Phys. 104, 1905-1913 (2006)

6. Mendolia, M.S., Farrington, G.C.: Ionic mobility in macromolecular electrolytes: The failure of Walden's rule. Chem. Mater. 5, 174-181 (1993)

7. Petrowsky, M., Frech, R.: Temperature dependence of ion transport: the compensated Arrhenius equation. J. Phys. Chem. B 113, 5996-6000 (2009)

8. Petrowsky, M., Frech, R.: Application of the compensated Arrhenius formalism to self-diffusion: implications for ionic conductivity and dielectric relaxation. J. Phys. Chem. B 114, 8600-8605 (2010)

9. Fleshman, A.M., Peterowsky, M., Jernigen, J.D., Bokalawela, R.S.P., Johnson, M.B., Frech, R.: Extending the compensated Arrhenius formalism to concentrated alcohol electrolytes: Arrhenius versus non-Arrhenius behavior. Electrochim. Acta 57, 147-152 (2011)

10. Bopege, D.N., Petrowsky, M., Fleshman, A.M., Frech, R., Johnson, M.B.: Temperature dependence of ion transport in dilute tetrabutylammonium triflate-acetate solutions and self-diffusion in pure acetate liquids. J. Phys. Chem. B 116, 71-76 (2012)

11. Stejskal, E.O., Tanner, J.E.: Spin diffusion measurements: spin echoes in the presence of a timedependent field gradient. J. Chem. Phys. 42, 288-292 (1965)

12. Bacelon, P., Corset, J., Loze, C.D.: Anion solvation II. Solvation of thiocyanate and halide anions in mixtures of protic and aprotic solvents. J. Solution Chem. 12, 13-22 (1983)

13. Bacelon, P., Corset, J., Loze, C.D.: Anion solvation III. Infrared spectroscopic determination of solvent acceptor numbers and their use in understanding anion solvation. J. Solution Chem. 12, 23-31 (1983)

14. Chakraborty, J.M., Das, B.: Electrical conductances and viscosities of tetrabutylammonium thiocyanate in acetonitrile in the temperature range $25-45^{\circ} \mathrm{C}$. Z. Phys. Chem. 218, 219-230 (2004)

15. Salomon, M.: Ion Association in 2-(chloromethyl)tetrahydrofuran. Electrochim. Acta 30, 1021-1026 (1985)

16. Petersen, G., Jacobsson, P., Torell, L.M.: A Raman study of ion-polymer and ion-ion interactions in low molecular weight polyether- $\mathrm{LiCF}_{3} \mathrm{SO}_{3}$ complexes. Electrochim. Acta 37, 1495-1497 (1992)

17. Schantz, S., Sandahl, J., Börjesson, L., Torell, L.M., Stevens, J.R.: Ion pairing in polymer electrolytes; A comparative Raman study of $\mathrm{NaCF}_{3} \mathrm{SO}_{3}$ complexed in poly(propylene-glycol) and dissolved in acetonitrile. Solid State Ionics 28-30, 1047-1053 (1988)

18. Schantz, S., Torell, L.M., Stevens, J.R.: Raman and Brillouin scattering of $\mathrm{LiClO}_{4}$ complexed in poly(propylene-glycol). J. Appl. Phys. 64, 2038-2043 (1988)

19. CRC Handbook of Chemistry and Physics, 79 edn. CRC Press, Boca Raton (1998-1999)

20. Pierce, L., Chang, C.K., Hayashi, M., Nelson, R.: Microwave spectrum, dipole moment and internal rotation of trans-2-butanone. J. Mol. Spec. 5, 449-457 (1969) 